and although none of the sandstones preserves any laminations or crossbedding typical of dune deposits, they are probably wind blown accumulations having advanced inland from the coast. The stones were presumably coughed up by the moas in the sandy coastal areas and buried by the advance of the dune sand.

The pebbles collected from Pukenuiorongo Bluffs are generally flatter and more rounded and polished than most of the authenticated moa gizzard stones which are usually found in association with moa skeletons. This supports Hayward's hypothesis that the collected pebbles were supplied by live moas who coughed them up when their grinding efficiency was significantly reduced. Hayward also proposed that the moas sought actual rock types even though the majority of the quartzose pebbles are gleaming white and easily distinguished the moas detected and swallowed numbers of brown, red and black pebbles of similar lithologies. It seems that the birds made their selection after a careful mineralogical assessment.

\section{Evidence for gluons?}

\section{from Frank Close}

THE latest results from PETRA, the Hamburg electron positron colliding ring, appear to add considerable weight to the quantum chromodynamic theory of the interquark force. Quarks are hypothesised to possess a new form of charge, known as colour, and interact with one another by exchanging coloured gluons (this process being somewhat analogous to the way that electrically charged objects mutually interact by exchanging photons). Just as the photon is the quantum of the electromagnetic force so are coloured gluons the quanta of the chromodynamic or interquark force. The theories of these forces are respectively quantum electrodynamics (QED) and quantum chromodynamics (QCD), the similarity in their names bearing witness to the similarity in their mathematical structures.

When electrons are annihilated by their antimatter (positrons) the energy is converted into light (this is well described by $Q E D)$. This light lives for only a fraction of a second before being transformed into particles. Sometimes the light reconverts into leptons and antileptons (like the electron and positron). Alternatively the light may become a quark and an antiquark ( $q$ and $\bar{q}$ ). This process is conventionally denoted

$$
\mathrm{e}^{+} \mathrm{e}^{-\rightarrow} \rightarrow \gamma \rightarrow \mathrm{q} \overline{\mathrm{q}}
$$

So far everything is described by QED. In both cases electrical charges are being accelerated (positive and negative have been produced out of nothing) and QED predicts that quanta (photons) will be radiated by the produced charged particles

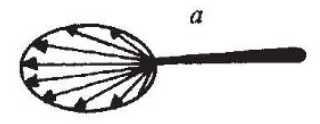

FAT PLUS THINJET
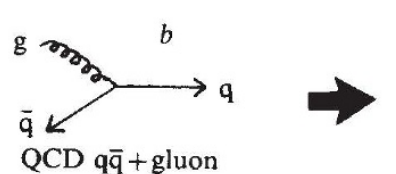

QCD $q \bar{q}+$ gluon

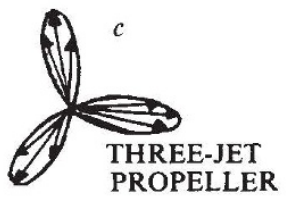

(the lepton pair or the quark and antiquark). This is indeed the case experimentally and has been well understood for many years.

QCD for colour carriers is analogous to QED for charge carriers. Therefore it may be no surprise to learn that when colour charges are accelerated the theory predicts that gluons will be radiated. Quarks and antiquarks carry colour and so the real process is

$$
\begin{aligned}
\mathrm{e}^{+} \mathrm{e}^{-} \rightarrow & \rightarrow \mathrm{r} \rightarrow(\mathrm{q}+\text { photons }+ \text { gluons })+ \\
& (\overline{\mathrm{q}}+\text { photons + gluons })
\end{aligned}
$$

From established QED we can calculate how much of the signal is due to the photon radiation. This then makes possible a study of the characteristics associated with gluon radiation from coloured quarks and comparison with the QCD predictions. There are three qualitative types of phenomena expected, one of which was only becoming apparent earlier this year (Nature 277, 349; 280, 450) and the third of which now appears to be showing up in the most recent Hamburg experiments.

(1) It is possible that the quark and antiquark may bind together and form a vector meson, like the $J / \psi$ or $I$ mesons, until such time as they annihilate one another thereby causing the meson to die. QCD predicts two things here. First, the probability for the quark and antiquark to annihilate decreases as the mass or energy of the $q \bar{q}$ bound state increases. This is apparently seen in tht the $\mathrm{J} / \psi(3.1 \mathrm{GeV})$ and $I(9.5 \mathrm{GeV})$ have anomalously enhanced lifetimes compared to what would have been expected in the absence of QCD based on the known lifetime of the lighter $\phi(1.0 \mathrm{GeV})$.

Second, it makes predictions as to the distribution in energy and space of the particles that are produced in the decay. The theory requires that the quark and antiquark annihilate, thereby producing three gluons. These gluons then transmute into pions and kaons that are detected by the experimental apparatus; three jets of particles should be detected bearing witness to the memory of the three gluons.

The $\mathrm{J} / \psi$ at $3 \mathrm{GeV}$ is too light to allow a good test of this prediction. Studies of the more massive $I$ early in 1979 began to provide data supporting the three-jet prediction (News and Views 277, 349; 280, 450). The best tests of this prediction may have to wait until (if?) a yet heavier vector meson $(\theta)$ is discovered, a bound state of the hypothesised sixth quark ( $t$ and $\bar{t})$. Such

F.E. Close is with the Theory Division, SRC Rutherford Laboratory. a meson, (if it exists) appears to be too massive to be produced at the present generation of electron-positron machines.

(2) The energy of the electron-positron annihilation might not be suitable for a vector meson to be simply produced. In this case the $q, \bar{q}$ and associated glue are radiated off away from one another.

QCD predicts that, at high energies, the most likely configurations are where the quark and antiquark carry off most of the energy and the glue is either very soft or highly collimated along the qq axis. As a consequence two jets of particles will be seen in the detectors.

Such 'two-jet events' have been known to exist for about 5 years. QCD predicts that as the electron-positron annihilation energies increase, so these two-jet events will become increasingly collimated and pencil-like. Data at the highest energies indeed support this.

(3) Although these jetlike - pencil events should dominate, there is a chance that the quark (or antiquark) radiates a hard gluon which carries off momentum and deflects the quark (antiquark) from its path (Fig 1a). If resolution is poor this will show up as a thin-jet plus fat-jet event, like a squash or tennis racket (Fig. 1a). Evidence for such events was reported by the TASSO collaboration at the CERN conference in June.

The latest results from PETRA are obtained with it operating at the highest energy of $32 \mathrm{GeV}$. The events are dominantly two-jet in character but a significant number of planar tennis racket type were seen. Some of these do appear to be resolvable into a genuine three-jet structure (Fig 1c) (known as propeller or Mercedes events - the German laboratory inspired by the manufacturers symbol). These latest results were announced at a conference at Fermilab, Chicago during the final week of August.

So it appears that, at least qualitatively, the behaviour of high energy electronpositron annihilation does mimic the expectations of $\mathrm{QCD}$, the theory of the interquark force. Theorists and experimentalists suspect that these threejet events are due to a quark radiating a gluon before the detected particles are created Figure $1 b$ shows the $\mathrm{q} \overrightarrow{\mathrm{q}}$ gluon state, which then becomes particles like pions and kaons by an unknown mechanism. However, there is much quantitative work still to be done by experimentatists, and theorists will be trying to find more precise tests. With more work at the highest energies, PETRA seems well set to be able to perform a significant test of the theory. 